\section{Atemwegsmanagement bei Wiederbelebung: Wann von Vorteil?}

Izawa J et al. Pre-hospital advanced airway management for adults with out-of-hospital cardiac arrest: nationwide cohort study. BMJ 2019; 364: 1430. doi:10.1136/bmj.1430

Erste-Hilfe-Maßnahmen, also Herzdruckmassage, Beatmung und ggf. eine Defibrillation, verbessern die Prognose bei einem Herzstillstand außerhalb der Klinik. Ausgebildete Rettungskräfte sind darüber hinaus in der Lage, ein geeignetes Atemwegsmanagement vorzunehmen. Ob die Vorteile dieser prästationären Maßnahmen zur Sicherung der Atemwege jedoch die Risiken aufwiegen, ist nicht sicher geklärt.

Wie steht es mit den Überlebenschancen von Patienten, die außerhalb der Klinik wiederbelebt werden müssen, abhängig davon, ob ein spezielles Atemwegsmanagement zur Verfügung steht oder nicht? Wem nützen supraglottische Atemwegshilfen bzw. eine endotracheale Intubation? Diesen Fragen gingen Izawa und Kollegen in einer Kohortenstudie nach. Sie werteten die Daten von 310620 Patienten eines nationalen Registers in Japan aus, die zwischen 2014 und 2016 außerhalb einer Klinik einen Herzstillstand erlitten hatten. In die Analyse schlossen sie die Daten der erwachsenen Personen ein, die vor Eintreffen der Rettungskräfte einen Herzstillstand erlitten hatten und entweder durch die Rettungskräfte selbst oder eine andere entsprechend zur Wiederbelebung ausgebildete Fachkraft reanimiert worden waren. Ausgeschlossen wurden Patienten über 120 Jahre, solche mit fehlenden Daten zur Reanimation sowie diejenigen, bei denen mehr als 30 Minuten zwischen dem Notruf und den Wiederbelebungsmaßnahmen vergangen waren.

Die Autoren unterteilten die Patienten je nach Ergebnis der ersten verfügbaren EKG-Aufzeichnung nach dem akuten Ereignis in 2 Gruppen: Bei insgesamt
20516 Patienten war ein Elektroschock indiziert (aufgrund von Kammerflimmern oder einer pulslosen ventrikulären Tachykardie); bei den übrigen 121890 bestand diese Indikation hingegen nicht (z. B. bei Asystolie). Als primären Endpunkt definierten die Autoren das Überleben nach einem Monat bzw. die Entlassung aus der Klinik.

Eine supraglottische Atemwegshilfe (z. B. Larynx-Tubus) oder endotracheale Intubation wurde bei 41,2\% der Gruppe mit Indikation für eine Defibrillation durchgeführt und bei $42 \%$ der übrigen Patienten. In einer ersten multivariaten Analyse ging das erweiterte Atemwegsmanagement bei allen Pateinten mit geringeren Überlebenschancen einher.

Für die genauere Beurteilung wurden die Patienten, bei denen im Rahmen der prästationären Reanimation ein Atemwegsmanagement erfolgte, anhand von Propensity-Score-Analysen mit solchen gematcht, die zum jeweils selben Zeitpunkt ( 0 bis 59 Minuten) diese Maßnahmen (noch) nicht erhalten hatten. Für diese Analyse ließen sich 16114 nach Elektroschock mit 236042 Patienten ohne Elektroschock matchen - die Mehrheit für die Zeitpunkte 5-10 Minuten nach Start der Reanimation. Hier zeigte sich: Bei den Patienten mit einer Indikation für einen Elektroschock war es für das Überleben unerheblich, ob ein Atemwegsmanagement erfolgt war oder nicht (19,2\% vs. 18,6\%; adjustierte Risk-Ratio 1,0; $95 \%$-Konfidenzintervall 0,93-1,07). Zudem waren die Patienten nach erfolgter Atemwegssicherung eher funktionell beeinträchtigt als die anderen. Anders verhielt es sich jedoch bei denjenigen ohne Indikation für eine Defibrillation: Hier ergaben sich mit 2,3\% vs. $1,8 \%$ deutlich höhere Überlebenschancen, wenn ein supraglottischer Tubus gelegt oder sogar endotracheal intubiert worden war (adjustierte Risk-Ratio 1,27; 95\%-Konfidenzintervall 1,2-1,35). Die spätere funktionelle Beeinträchtigung wurde hier durch das jeweilige Vorgehen nicht beeinflusst.
FAZIT

Offenbar ist das Vorliegen einer Indikation für einen Elektroschock entscheidend für das weitere Vorgehen bei einem Herzstillstand außerhalb der Klinik, so die Autoren: Ist ein Elektroschock sinnvoll, sollten die Defibrillation und Herzdruckmassage im Vordergrund stehen; eine erweiterte Atemwegsicherung erscheint nachrangig. Weist das EKG hingegen nicht auf die Option eines Elektroschocks hin, sei vermutlich die Sicherstellung Sauerstoffzufuhr durch geeignete Maßnahmen vorrangig.

Dr. med. Susanne Meinrenken, Bremen 